\title{
Prostatic Urethral Malignant Neoplasm
}

National Cancer Institute

\section{Source}

National Cancer Institute. Prostatic Urethral Malignant Neoplasm. NCI Thesaurus. Code C39870.

A primary or metastatic malignant neoplasm that affects the prostatic urethra. 